\title{
Pacific
}

Journal of

Mathematics

\section{DISCRETE MORSE THEORY AND HOPF BUNDLES}

DMITRY N. KOZLOV 


\title{
DISCRETE MORSE THEORY AND HOPF BUNDLES
}

\author{
DMITRY N. KOZLOV
}

\begin{abstract}
We use Hopf bundles to give an example of a regular CW complex $X$ and an acyclic matching $M$ on the face poset of $X$, such that there are no critical cells in neighboring dimensions but the complex $X$ is not homotopy equivalent to the corresponding wedge of spheres. The key fact here is that the higher homotopy groups of spheres are nontrivial. We also give a sufficient condition on an acyclic matching $M$ for concluding that $X$ is homotopy equivalent to a wedge of spheres indexed by the critical cells.
\end{abstract}

\section{Introduction}

Discrete Morse theory, introduced by Robin Forman [1998], has become quite a useful tool for doing specific computations in combinatorial algebraic topology; see [Kozlov 2008] for the general framework, and [Clark and Ehrenborg $\geq 2011$ ] for an interesting recent application in case of the Frobenius complex.

Let us briefly describe how the computational model provided by discrete Morse theory works. Given a regular CW complex $X$, let $\mathscr{F}(X)$ denote the poset of all nonempty cells of $X$. This poset is ranked by the dimensions of the cells. A partial matching on the Hasse diagram of $\mathscr{F}(X)$ is a bijection $M: U \rightarrow D$, where $U$ and $D$ are (possibly empty) disjoint sets of elements of $\mathscr{F}(X)$ such that $\operatorname{dim}(\sigma)=\operatorname{dim}(M(\sigma))+1$, and $M(\sigma)$ lies on the boundary of $\sigma$ for all $\sigma \in U$. A partial matching $M$ is called acyclic if there do not exist $\sigma_{1}, \ldots, \sigma_{t} \in \mathscr{F}(X)$ such that $\sigma_{1} \neq \cdots \neq \sigma_{t}$, and $\sigma_{i+1}>M\left(\sigma_{i}\right)$ for all $i=1, \ldots, t$, where as usual we set $\sigma_{t+1}:=\sigma_{1}$. We set $C_{M}:=\mathscr{F}(X) \backslash(U \cup D)$ and call the elements of $C$ critical. For all $i$, let $f_{i}\left(C_{M}\right)$ denote the number of critical cells of dimension $i$. The main theorem in [Forman 1998] states that whenever $M$ is an acyclic matching, there exists a CW complex $\widetilde{X}$, called the critical Morse complex, with $f_{i}\left(C_{M}\right)$ cells of dimension $i$, for all $i$, such that $\tilde{X}$ is homotopy equivalent to $X$.

Frequently, the actual goal of applying discrete Morse theory is to prove that $X$ is in fact homotopy equivalent to a wedge of spheres, or at least to compute the

This research was supported by University of Bremen, as part of AG CALTOP.

MSC2000: primary 57Q05; secondary 54G20.

Keywords: homotopy group, fibrations, gluing map, acyclic matching, long exact sequence for homotopy. 
homology groups of $X$. In a very fortunate situation, one might be able to produce an acyclic matching $M$ such that for all $i$ we have $f_{i}\left(C_{M}\right) f_{i+1}\left(C_{M}\right)=0$, that is, there are no critical cells in neighboring dimensions. This would settle the question of computing the homology groups of the space $X$. However, in order to determine the homotopy type of $X$, one would want to conclude that the critical cells are somehow independent of each other, and so we have the homotopy equivalence

$$
X \simeq \bigvee_{i} \underbrace{S^{i} \vee \cdots \vee S^{i}}_{\tilde{f}_{i}\left(C_{M}\right)},
$$

where $\tilde{f}_{i}\left(C_{M}\right)=f_{i}\left(C_{M}\right)$ for $i \geq 1$ and $\tilde{f}_{0}\left(C_{M}\right)=f_{0}\left(C_{M}\right)-1$, and we use the convention that the empty wedge is a point.

We will use the fact that higher homotopy groups of spheres are nontrivial to give an example showing that just assuming that there are no critical cells in neighboring dimensions is not enough to conclude that the space is homotopy equivalent to a wedge of spheres.

But first, on the positive side, we give a sufficient condition on acyclic matching that lets us conclude that the space is homotopy equivalent to a wedge of spheres indexed by the critical cells. In fact, for this result we will not need the condition that there are no critical cells in neighboring dimensions; see also [Clark and Ehrenborg $\geq 2011$, Theorem 2.5].

\section{Acyclic matchings yielding a wedge of spheres}

Here we are interested in acyclic matchings that allow us to conclude that the considered complex is in fact homotopy equivalent to a wedge of spheres that are enumerated by the critical cells. First we need some terminology.

Definition 2.1. Let $P$ be a partially ordered set and $M$ a partial matching on $P$.

(1) A generalized alternating path is a sequence $x_{1}>x_{2}<x_{3}>\cdots<x_{2 t+1}$ or a sequence $x_{1}>x_{2}<x_{3}>\cdots>x_{2 t+2}$, where $t \geq 0$, such that $M\left(x_{2 k+1}\right)=x_{2 k}$ for all $k=1, \ldots, t$.

(2) Let $x$ be an element of $P$. We set $F(x)$ to be the set of the endpoints of all generalized alternating paths starting at $x$, and call $F(x)$ the feasibility domain of $x$.

Note that in a generalized alternating path, we require that $x_{2 k+1}$ covers $x_{2 k}$ for all $k=1, \ldots, t$, but we obviously do not require that $x_{2 k-1}$ covers $x_{2 k}$ for all such $k$.

It is easy to see that $F(x)$ shall always contain a critical cell of dimension 0 . Let $A$ denote the set of 0 -dimensional cells in $F(x)$. If none of them is critical, then there exists the set of 1-dimensional cells $B \subset F(x)$ such that $M: B \rightarrow A$ is a bijection. Since every $y \in B$ covers two elements, the graph with the vertex set 
$A \cup B$ and the covering relations as edges cannot be a forest, so it contains cycles, which contradicts the assumption that the matching $M$ is acyclic.

The following theorem gives a sufficient condition on an acyclic matching for the critical Morse complex to be homotopy equivalent to a wedge of spheres enumerated by critical cells.

Theorem 2.2. Let $X$ be a connected regular $C W$ complex, and let $M$ be an acyclic partial matching on $\mathscr{F}(X)$. Assume that for every critical cell $c$ of dimension larger than 0 , its feasibility domain $F(c)$ contains precisely two critical cells: $c$ itself and one critical cell of dimension 0 . Then $X$ is homotopy equivalent to a wedge of spheres enumerated by critical cells, that is, (1-1) is true.

Proof. For this argument, we adopt the point of view of [Kozlov 2008] and follow the proof of its Theorem 11.13(b). There the main theorem of discrete Morse theory for CW complexes is proved by a stepwise attachment of either a critical cell or of a pair of cells matched by $M$, with a parallel explicit construction of a Morse homotopy map. This stepwise attachment is done along a certain linear extension of the face poset of $X$, which we denote by $l$. When a pair of matched cells is attached, we simply have a strong deformation retraction of the obtained complex to what we have had before that attachment, so we just need to understand the case of attaching a critical cell.

Assume that a critical cell $c$ of dimension at least 1 is being attached. The cells in $F(c) \backslash\{c\}$ form a subcomplex $C$ of $X$. The assumption of the theorem implies that $C$ is collapsible along the matching $M$. It means that prior to the attachment of $c$, the Morse homotopy has already shrunk the complex $C$ to a point $a$, where $a$ is the critical 0 -dimensional cell of $F(c) \backslash\{c\}$. Since the image of the attaching map of the cell $c$ lies inside $C$, we conclude that in the critical Morse complex, the attaching map of $c$ will simply map everything to the point $a$. Thus we can conclude that all the attaching maps in the critical Morse complex are trivial.

Finally, we need to see that the matching $M$ has exactly one 0-dimensional critical cell, which will imply that all the critical cells will be attached to the same vertex. Assume we have another critical 0 -dimensional cell $b$, and assume that $b$ occurs after $a$ in the linear extension $l$. Then, when $b$ is added, it will form a new connected component. So, since the total complex $X$ is connected, at some point in the inductive process of adding critical cells and pairs of matched cells we will have to connect that connected component to the connected component containing $a$. This can only be achieved by adding a critical 1-dimensional cell, which we call $e$. The set $F(e)$ cannot contain any critical 0 -dimensional cells other than $b$. Let $v_{1}$ denote the vertex of $e$ that does not lie in the same connected component as $b$. The vertex $v_{1}$ is not critical, and we set $e_{1}:=M^{-1}\left(v_{1}\right)$. Both $v_{1}$ and $e_{1}$ were added before $e$. We now proceed, starting with $k=1$ be letting 

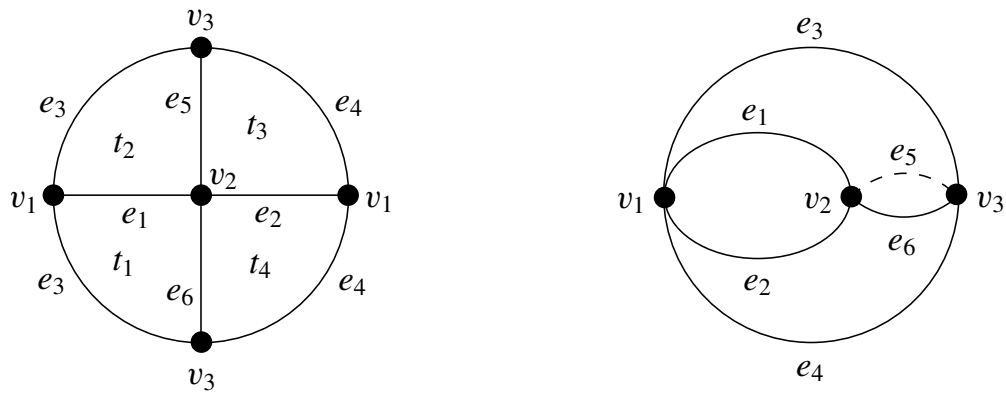

Figure 2.1. Two presentations of the simplicial complex $L$.

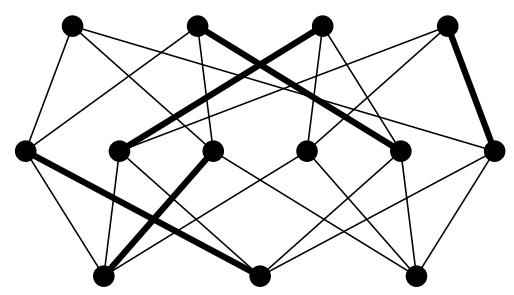

Figure 2.2. The face poset of $L$. The vertices, edges, and triangles are shown with the index increasing from left to right. The bold edges indicate an acyclic matching.

$v_{k+1}$ be the vertex of $e_{k}$ other than $v_{k}$. Since $v_{k+1} \in F(e)$, we see that $v_{k+1}$ is not critical, and set $e_{k+1}:=M^{-1}\left(v_{k+1}\right)$. Both $v_{k+1}$ and $e_{k+1}$ were added before $e$. Eventually we will have to conclude that for some $k \geq 1$ the vertex $v_{k+1}$ lies in the same connected component as $b$. But this means that $b$ was connected to the vertex $v_{1}$ even before adding $e$, yielding a contradiction to the choice of $e$.

The condition of Theorem 2.2 is not necessary for getting a wedge of spheres enumerated by critical cells. For example, let $L$ be the simplicial complex shown on Figure 2.1. A direct examination yields that the matching shown on Figure 2.2 is acyclic with one critical cell in each of the dimensions 0,1 , and 2 . The condition of Theorem 2.2 is not satisfied, but the space $L$ is homotopy equivalent to $S^{1} \vee S^{2}$.

\section{Hopf fiber bundles}

We now give an example that simply having an acyclic matching with no critical cells in neighboring dimensions is not sufficient to conclude that the space $X$ is homotopy equivalent to a wedge of spheres. Our example exploits the fact that the higher homotopy groups (unlike the homology groups) of spheres are nontrivial. The first such nontrivial group is $\pi_{3}\left(S^{2}\right)=\mathbb{Z}$, and it is this one which we use for our construction. 
Consider the set $A:=\left\{\left.\left(z_{1}, z_{2}\right)\left|z_{1}, z_{2} \in \mathbb{C},\right| z_{1}\right|^{2}+\left|z_{2}\right|^{2}=1\right\}$, and let the multiplicative group $G=\{z|| z \mid=1\} \subseteq \mathbb{C}$ act on $A$ diagonally by multiplication: $z:\left(z_{1}, z_{2}\right) \mapsto\left(z z_{1}, z z_{2}\right)$. The quotient $A / G$ can be viewed as a complex projective line $\mathbb{C P}^{1}$, with the quotient map $q: A \rightarrow A / G$ simply being $q:\left(z_{1}, z_{2}\right) \mapsto\left(z_{1}: z_{2}\right)$. Note that topologically $A \cong S^{3}, G \cong S^{1}$, and $A / G \cong S^{2}$. The obtained fiber bundle $S^{1} \rightarrow S^{3} \rightarrow S^{2}$ is the first example of a Hopf bundle; see [Hatcher 2002, Example 4.45].

Consider the $\mathrm{CW}$ structure on $A$ obtained by intersecting with the real coordinate hyperplanes $\operatorname{Re} z_{1}=0, \operatorname{Im} z_{1}=0, \operatorname{Re} z_{2}=0$, and $\operatorname{Im} z_{2}=0$. Then $A$ is a regular $\mathrm{CW}$ complex with face vector $(8,24,32,16)$. Furthermore, consider the $\mathrm{CW}$ structure on $A / G$ consisting of the two vertices $v_{1}=(1: 0)$ and $v_{2}=(0: 1)$, four edges $e_{1}=\{(1: r) \mid r>0\}, e_{2}=\{(1: i r) \mid r>0\}, e_{3}=\{(1:-r) \mid r>0\}$, and $e_{4}=\{(1:-i r) \mid r>0\}$, and four 2-cells denoted $s_{1}, s_{2}, s_{3}, s_{4}$, where $s_{i}$ is bound by $e_{i}$ and $e_{i+1}$ for $i=1,2,3$, and $s_{4}$ is bound by $e_{1}$ and $e_{4}$. Again $A / G$ is a regular $\mathrm{CW}$ complex with the face vector $(2,4,4)$, and one sees that $q$ is a cellular map.

Set $C:=((A \times[0,1]) \bigsqcup(A / G)) / \sim$ to be the mapping cylinder of $q$, that is $\sim$ is given by $(a, 1) \sim q(a)$, for all $a \in A$. We choose a $C W$ structure on $C$ by taking all the cells of $A / G \subseteq C$, subdividing $A \times\{0\}$, the top copy of $A$, as described above, and taking the open cells $\tilde{\sigma}:=\operatorname{int} \sigma \times(0,1)$ for all cells $\sigma$ of $A$. Here we write int $\sigma=\sigma \backslash \partial \sigma$ to denote the relative interiors of cells. Finally, let $X$ be the regular CW complex obtained from $C$ by attaching a 4-cell $k$ along $A \times\{0\} \cong S^{3}$.

Consider the following acyclic matching: $M(\tilde{\sigma})=\sigma$ whenever $\sigma$ is a cell of $A \times\{0\}, M\left(s_{i}\right)=e_{i}$ for $i=1,2,3$, and $M\left(e_{4}\right)=v_{2}$. The partial matching $M$ has three critical cells: $v_{1}, k$, and $s_{4}$, in dimensions 0,2 , and 4 . It is easily verified directly that all the matched pairs are regular in the sense of [Cohen 1973] and [Kozlov 2008, Definition 11.12]; in particular the main theorem of discrete Morse theory (see [Forman 1998]) can be applied and we can conclude that $X$ is homotopy equivalent to a $\mathrm{CW}$ complex with one cell in each of the dimensions 0,2 , and 4 .

However, the space $X$ is not homotopy equivalent to $S^{2} \vee S^{4}$. For example, these two spaces have different $\pi_{3}$ groups. ${ }^{1}$ Namely $\pi_{3}(X)=0$, while $\pi_{3}\left(S^{2} \vee S^{4}\right)=\mathbb{Z}$. Both of these statements can be seen using the long exact sequence for relative homotopy; see [Hatcher 2002, page 344]. Indeed, when a space $\widetilde{Y}$ is obtained from a space $Y \cong S^{2}$ by attaching a 4-cell along some continuous map $\varphi: S^{3} \rightarrow S^{2}$, the relevant part of the long exact sequence for homotopy of the pair $(\tilde{Y}, Y)$ is

$$
\cdots \rightarrow \pi_{4}(\tilde{Y}, Y, y) \stackrel{\partial}{\longrightarrow} \pi_{3}(Y, y) \stackrel{i_{*}}{\longrightarrow} \pi_{3}(\tilde{Y}, y) \rightarrow \pi_{3}(\tilde{Y}, Y, y) \rightarrow \cdots,
$$

${ }^{1}$ Serge Ochanine pointed out to the author in 2009 that the constructed space $X$ is actually the complex projective plane. We use the long exact sequence of the homotopy of a pair to show how we came up with the example. 
where $y \in Y$ is a base point, the map $\partial$ comes from restricting maps $\left(D^{n}, S^{n-1}, s\right) \rightarrow$ $(\tilde{Y}, Y, y)$ to $S^{n-1}$, and $i_{*}$ is the map between homotopy groups induced by the inclusion map $i: Y \hookrightarrow \widetilde{Y}$. Since $(Y, y) \cong\left(S^{2}, x_{2}\right)$, and $(\tilde{Y}, Y, y) \simeq\left(D^{4}, S^{3}, x_{3}\right)$, where $x_{2} \in S^{2}$ and $x_{3} \in S^{3}$ are corresponding base points, the sequence (3-1) translates to

$$
\cdots \rightarrow \pi_{3}\left(S^{3}, x_{3}\right) \stackrel{\varphi_{*}}{\longrightarrow} \pi_{3}\left(S^{2}, x_{2}\right) \rightarrow \pi_{3}(\tilde{Y}, y) \rightarrow 0 \rightarrow \cdots .
$$

For the space $S^{2} \vee S^{4}$ the map $\varphi_{*}$ is trivial, and hence $\pi_{3}(\tilde{Y}, y)=\pi_{3}\left(S^{2}, x_{2}\right)=\mathbb{Z}$; for the case of the Hopf bundle above, the map $\varphi_{*}$ is surjective, and so we get $\pi_{3}(\tilde{Y}, y)=0$. Clearly, this technique can be used to produce further examples that might be needed to test various hypothesis.

Taking the barycentric subdivision of $X$ will yield a simplicial complex with the same property: It has an acyclic matching with one critical cell of dimensions 0,2 , and 4 each, but is of course homeomorphic to the regular CW version; in particular, it is not homotopy equivalent to $S^{2} \vee S^{4}$. We leave finding such an acyclic matching to the reader.

\section{Acknowledgments}

I thank the University of Bremen and the Mathematical Sciences Research Institute at Berkeley for being able to conduct this work. I also thank Eric Clark, Richard Ehrenborg, and Serge Ochanine for their valuable comments.

\section{References}

[Clark and Ehrenborg $\geq 2011]$ E. Clark and R. Ehrenborg, "The Frobenius complex", preprint. To appear in Ann. Comb.

[Cohen 1973] M. M. Cohen, A course in simple-homotopy theory, Graduate Texts in Mathematics 10, Springer, New York, 1973. MR 50 \#14762 Zbl 0261.57009

[Forman 1998] R. Forman, "Morse theory for cell complexes", Adv. Math. 134:1 (1998), 90-145. MR 99b:57050 Zbl 0896.57023

[Hatcher 2002] A. Hatcher, Algebraic topology, Cambridge University Press, Cambridge, 2002. MR 2002k:55001 Zbl 1044.55001

[Kozlov 2008] D. Kozlov, Combinatorial algebraic topology, Algorithms and Computation in Mathematics 21, Springer, Berlin, 2008. MR 2008j:55001 Zbl 1130.55001

Received November 12, 2009.

DMITRY N. KOZLOV

DEPARTMENT OF MATHEMATICS

BREMEN UNIVERSITY

BIBLIOTHEKSSTRASSE 1

D-28334 BREMEN

GERMANY

dfk@math.uni-bremen.de

http://www.math.uni-bremen.de/ dfk/ 


\title{
PACIFIC JOURNAL OF MATHEMATICS
}

\author{
http://www.pjmath.org \\ Founded in 1951 by
}

E. F. Beckenbach (1906-1982) and F. Wolf (1904-1989)

\section{EDITORS}

V. S. Varadarajan (Managing Editor)

Department of Mathematics

University of California

Los Angeles, CA 90095-1555

pacific@math.ucla.edu

Vyjayanthi Chari

Department of Mathematics

University of California

Riverside, CA 92521-0135

chari@math.ucr.edu

\section{Robert Finn}

Department of Mathematics Stanford University

Stanford, CA 94305-2125

finn@math.stanford.edu

Kefeng Liu

Department of Mathematics

University of California

Los Angeles, CA 90095-1555

liu@math.ucla.edu
Darren Long

Department of Mathematics

University of California

Santa Barbara, CA 93106-3080

long@math.ucsb.edu

Jiang-Hua Lu

Department of Mathematics

The University of Hong Kong

Pokfulam Rd., Hong Kong jhlu@maths.hku.hk

Alexander Merkurjev

Department of Mathematics University of California

Los Angeles, CA 90095-1555 merkurev@math.ucla.edu
Sorin Popa

Department of Mathematics

University of California

Los Angeles, CA 90095-1555

popa@math.ucla.edu

Jie Qing

Department of Mathematics

University of California

Santa Cruz, CA 95064

qing@ cats.ucsc.edu

Jonathan Rogawski

Department of Mathematics

University of California

Los Angeles, CA 90095-1555

jonr@math.ucla.edu

\section{PRODUCTION}

pacific@math.berkeley.edu

Silvio Levy, Scientific Editor Matthew Cargo, Senior Production Editor

ACADEMIA SINICA, TAIPEI

CALIFORNIA INST. OF TECHNOLOGY

INST. DE MATEMÁTICA PURA E APLICADA

KEIO UNIVERSITY

MATH. SCIENCES RESEARCH INSTITUTE

NEW MEXICO STATE UNIV.

OREGON STATE UNIV.

\section{SUPPORTING INSTITUTIONS}

STANFORD UNIVERSITY
UNIV. OF BRITISH COLUMBIA
UNIV. OF CALIFORNIA, BERKELEY
UNIV. OF CALIFORNIA, DAVIS
UNIV. OF CALIFORNIA, LOS ANGELES
UNIV. OF CALIFORNIA, RIVERSIDE
UNIV. OF CALIFORNIA, SAN DIEGO
UNIV. OF CALIF., SANTA BARBARA

UNIV. OF CALIF., SANTA CRUZ

UNIV. OF MONTANA

UNIV. OF OREGON

UNIV. OF SOUTHERN CALIFORNIA

UNIV. OF UTAH

UNIV. OF WASHINGTON

WASHINGTON STATE UNIVERSITY

These supporting institutions contribute to the cost of publication of this Journal, but they are not owners or publishers and have no responsibility for its contents or policies.

See inside back cover or www.pjmath.org for submission instructions.

The subscription price for 2011 is US \$420/year for the electronic version, and \$485/year for print and electronic.

Subscriptions, requests for back issues from the last three years and changes of subscribers address should be sent to Pacific Journal of Mathematics, P.O. Box 4163, Berkeley, CA 94704-0163, U.S.A. Prior back issues are obtainable from Periodicals Service Company, 11 Main Street, Germantown, NY 12526-5635. The Pacific Journal of Mathematics is indexed by Mathematical Reviews, Zentralblatt MATH, PASCAL CNRS Index, Referativnyi Zhurnal, Current Mathematical Publications and the Science Citation Index.

The Pacific Journal of Mathematics (ISSN 0030-8730) at the University of California, c/o Department of Mathematics, 969 Evans Hall, Berkeley, CA 94720-3840, is published monthly except July and August. Periodical rate postage paid at Berkeley, CA 94704, and additional mailing offices. POSTMASTER: send address changes to Pacific Journal of Mathematics, P.O. Box 4163, Berkeley, CA 94704-0163.

PJM peer review and production are managed by EditFLOW ${ }^{\mathrm{TM}}$ from Mathematical Sciences Publishers.

PUBLISHED BY PACIFIC JOURNAL OF MATHEMATICS

at the University of California, Berkeley 94720-3840

A NON-PROFIT CORPORATION

Typeset in IATEX

Copyright $(2011$ by Pacific Journal of Mathematics 


\section{PACIFIC JOURNAL OF MATHEMATICS}

Volume $249 \quad$ No. $2 \quad$ February 2011

A gluing construction for prescribed mean curvature

257

ADRIAN BUTSCHER

Large eigenvalues and concentration

271

BRUNO COLBOIS and ALESSANDRO SAVO

Sur les conditions d'existence des faisceaux semi-stables sur les courbes multiples primitives

JEAN-MARC DRÉZET

A quantitative estimate for quasiintegral points in orbits

LIANG-CHUNG HSIA and JosePh H. Silverman

Möbius isoparametric hypersurfaces with three distinct principal curvatures, II

ZEJUN HU and SHUJIE ZHAI

Discrete Morse theory and Hopf bundles

371

DMITRY N. KOZLOV

Regularity of canonical and deficiency modules for monomial ideals

ManOJ KUMmini and SATOSHI MURAI

$\mathrm{SL}_{2}(\mathbb{C})$-character variety of a hyperbolic link and regulator

WEIPING LI and QINGXUE WANG

Hypergeometric evaluation identities and supercongruences

LING LONG

Necessary and sufficient conditions for unit graphs to be Hamiltonian

H. R. Maimani, M. R. Pournaki and S. Yassemi

Instability of the geodesic flow for the energy functional

DOMENico PERrone

String structures and canonical 3-forms

CORBETT REDDEN

Dual pairs and contragredients of irreducible representations

BINYONG SUN

On the number of pairs of positive integers $x_{1}, x_{2} \leq H$ such that $x_{1} x_{2}$ is a $k$-th power

DOYCHIN I. TOLEV

Correction to the article A Floer homology for exact contact embeddings 